Proyecciones Journal of Mathematics

Vol. 36, No 2, pp. 225-244, June 2017.

Universidad Católica del Norte

Antofagasta - Chile

\title{
A brief note on the existence of connections and covariant derivatives on modules
}

\author{
Jacqueline Rojas \\ Universidade Federal da Paraiba, Brasil \\ and \\ Ramón Mendoza \\ Universidade Federal de Pernambuco, Brasil \\ Received : February 2016. Accepted : March 2017
}

\begin{abstract}
In this note we make a review of the concepts of connection and covariant derivative on modules, in a purely algebraic context. Throughout the text, we consider algebras over an algebraically closed field of characteristic 0 and module will always mean left module. First, we concentrate our attention on a $k$-algebra $A$ which is commutative, and use the Kähler differentials module, $\Omega_{A / k}^{1}$, to define connection (see Subsection 2.1). In this context, it is verified that the existence of connections implies the existence of covariant derivatives (cf. Prop. 2.3), and that every projective module admits a connection (cf. Prop. 2.5). Next (in Section 3), we focus our attention in the discussion of some counterexamples comparing these two notions. In fact, it is known that these two notions are equivalent when we consider regular $k$-algebras of finite type (see [18], Prop. 4.2). As well as, that there exists a connection on $M$ if, and only if, the Atiyah-Kodaira-Spencer class of $M, c(M)$, is zero (see [17], Prop. 4.3). Finally, we take into account the case where $A$ is (not necessarily commutative) and it is used the bimodule, $\Omega^{1}$, of noncommutative differentials introduces by Connes ([9], [10]) in place of Kähler differentials to define a connection. In this case, it is proven that a module admits such connection if, and only if, it is a projective module (see [25], Theorem 5.2).
\end{abstract}

MSC 2010 : Primary 13C05; Secondary $13 N 05$.

Key words : Connection, covariant derivative, projective module. 


\section{Introduction}

The notions of connection and covariant derivative come from Riemannian geometry, where there is no distinction between them (just different points of view). We begin by doing a brief historical review of these notions.

In 1869 the German mathematician and physicist Elwin Bruno Christoffel (1829-1900) published Über die Transformation der homogenen Differentialausdrücke zweiten Grades in which he introduced the famous symbols

$\Gamma_{i j}^{k}$ (Christoffel's notation was $\left.\left\{\begin{array}{c}k \\ i j\end{array}\right\}\right)([8])$. What Christoffel did not realize was the fact that the symbols he had discovered themselves determine a connection on the $\mathcal{C}_{\mathcal{U}}^{\infty}$-module of sections of the tangent bundle, $\Gamma\left(T_{\mathcal{U}}\right)\left(\mathcal{C}_{\mathcal{U}}^{\infty}\right.$ denote the $\mathbf{R}$-algebra of all infinitely differentiable real-valued functions on the differential manifold $\mathcal{U}$ ).

It was noticed after by the Italian mathematicians Gregorio RicciCurbastro (1853-1925) and Tullio Levi-Civita (1873-1941) (in [26]) that the Christoffel symbols obtained from the Riemannian metric could be used to create a "coordinate free" differential calculus. This extension of the Differential Calculus allowed a modification of partial differentiation to spaces with Riemannian metric (i.e. non-Euclidean) by means of a covariant derivative.

In fact, they defined the covariant derivative of a vector field $X$ ( $X=$ $\sum_{j=1}^{n} X^{j} \frac{\partial}{\partial x_{j}}$ in local coordinates) to be: An $\mathbf{R}$-linear endomorphism of $\Gamma\left(T_{\mathcal{U}}\right)$ (the sections of the tangent bundle associated to the $n$-dimensional differential manifold $\mathcal{U}$ ) defined locally by the endomorphisms $\nabla_{1}, \ldots, \nabla_{n}$ where the $k$-th component of $\nabla_{i} X$ is given by $\left(\nabla_{i} X\right)^{k}=\frac{\partial X^{k}}{\partial x_{i}}+\sum_{j=1}^{n} X^{j} \Gamma_{i j}^{k}$. In the Euclidean space $\mathbf{R}^{n}$ with the usual Riemannian metric, the sum is zero (since all the Christoffel symbols are zero) and hence the covariant derivative is just an ordinary partial derivative.

In the 1920's the French mathematician Élie Joseph Cartan (1869-1951) developed a new notion of connection, the projective and conformal connections (see [5] and [6]).

In 1950 the French mathematician Jean-Louis Koszul gave an algebraic framework for regarding a connection as a differential operator. The Koszul connection was both more general than that of Levi-Civita, and was easier to work with because it finally was able to eliminate (or at least to hide) the awkward Christoffel symbols from the connection formalism ([24]).

It was Cartan's student Charles Ehresmann (1905-1979) who finally untangled and successfully classified all of the generalized and specific connections which had emerged in the first half of the 20th century $([15])$. He 
began this endeavor in an effort to understand Cartan's connections from a global point of view. Toward this end Ehresmann introduced the concept of fiber bundles (independently of Whitney and Steenrod). Ehresmann published his first notes on the subject during the period 1941-1944 in which he defines locally trivial principal bundles and their associated fiber bundles (also locally trivial). In his 1943 paper Sur les espaces fibrés associés une variété différentiable a manifold is defined by means of an atlas of local charts for the first time ([14]).

For example, in [4] an (affine) connection on the differential manifold $\mathcal{U}$ is defined as a mapping $\mathbf{D}: \Gamma\left(T_{\mathcal{U}}\right) \times \Gamma\left(T_{\mathcal{U}}\right) \rightarrow \Gamma\left(T_{\mathcal{U}}\right)$ where $\mathbf{D}(X, Y)=$ $\mathbf{D}_{X}(Y)$ satisfy the following three properties for every $f \in \mathcal{C}_{\mathcal{U}}^{\infty}$ and $X, Y, Z \in$ $\Gamma\left(T_{\mathcal{U}}\right)$ :

$$
\begin{gathered}
\mathbf{D}_{f X+Y}(Z)=f \mathbf{D}_{X}(Z)+\mathbf{D}_{Y}(Z), \\
\mathbf{D}_{X}(Y+Z)=\mathbf{D}_{X}(Y)+\mathbf{D}_{X}(Z) \\
\mathbf{D}_{X}(f Y)=f \mathbf{D}_{X}(Y)+X(f) Y .
\end{gathered}
$$

Since any vector field can be considered as a derivation in $\mathcal{C}_{\mathcal{U}}^{\infty}$, i.e. $\Gamma\left(T_{\mathcal{U}}\right)=\mathcal{D}_{\text {er }}\left(\mathcal{C}_{\mathcal{U}}^{\infty}\right)\left(\right.$ cf. [22]). So $\mathbf{D}$ above can be thought as an $\mathcal{C}_{\mathcal{U}}^{\infty}$-linear homomorphism from $\mathcal{D}_{\text {er }}\left(\mathcal{C}_{\mathcal{U}}^{\infty}\right)$ to $\operatorname{End}_{\mathbf{R}}\left(\Gamma\left(T_{\mathcal{U}}\right)\right)$ satisfying the third condition. Thus, it is very natural to study this notion in a purely algebraic context, we will do that in Subsection 2.1, where we call covariant derivative such kind of homomorphism (see also [20] and [23]). In particular, we can consider covariant derivatives for other vector bundles $E$ on $\mathcal{U}$. Related to this see example 2.6.

On the other hand, the notion of connection has appears in physics from time to time. For example, in 1954 the Chinese physicist Chen-Ning Franklin Yang (1922) and the American physicist Robert L. Mills (19271999) proposed a Gauge theory that allows us to understand the interaction proton-neutron. In their mathematical formulation, appear the now called Yang-Mills functional, which it is defined over the set of connection of a fix vector bundle. For example, if you consider the trivial complex vector bundle $\mathbf{R}^{4} \times \mathbf{C} \rightarrow \mathbf{R}^{4}$ with a given hermitian product on its sections, then we can deduce the Maxwell's equations of electrodynamics ([28]).

However the notion of connection reappears again in the context of noncommutative geometry $([9],[10])$. In fact, Connes introduced the notion of noncommutative differentials, which was used in place of Kähler differentials (in the definition of connection given in Subsection 2.1) by Krämer (in [25]), to conclude that the existence of such connections, it is verified exactly for projective modules over not (necessarily) commutative rings (cf. Theorem 5.2). 


\section{Connection and Covariant Derivative over modules}

Let $k$ be an algebraically closed field of characteristic $0, A$ a commutative $k$-algebra, and $M$ an $A$-module (module means left module).

A derivation from $A$ to $M$ is a $k$-linear map $\partial: A \rightarrow M$ such that $\partial(a b)=a \partial(b)+b \partial(a)$ for every $a, b \in A$.

One can readily verify under this definition that $\partial(1)=0$ and hence $\partial(r)=0$ for any $r \in k$.

Set $\mathcal{D}_{\mathrm{er}}(A, M)$ be the $A$-module of derivation from $A$ to $M$ and $\mathcal{D} \operatorname{er}(A)=$ $\mathcal{D}_{\text {er }}(A, A)$.

The Kähler differential module, $\Omega_{A / k}^{1}$. The module of Kähler differential is an $A$-module $\Omega_{A / k}^{1}$ together with an universal derivation $d: A \rightarrow \Omega_{A / k}^{1}$ which is universal in the following sense: for any $A$-module $M$, for any derivation $\partial \in \mathcal{D}_{\text {er }}(A, M)$, there exists a unique $A$-module homomorphism $\hat{\partial}: \Omega_{A / k}^{1} \longrightarrow M$ such that $\hat{\partial} \circ d=\partial$.

2.1. Example. Let $A=k\left[x_{1}, \ldots, x_{n}\right]$ be the ring of polynomials in $n$ variables. Then

- $\mathcal{D}_{\mathrm{er}}(A)$ is a free $A$-module with basis $\left\{\partial_{1}, \ldots, \partial_{n}\right\}$ (here $\partial_{i}=\frac{\partial}{\partial x_{i}}$ for $i=1, \ldots n)$.

- Follows from the universal property that $\Omega_{A / k}^{1} \cong A^{n}$ with $d x_{i}=e_{i}$ for $i=1, \ldots, n$ ( $\left\{e_{i}\right\}$ is the canonical basis of the free $A$-module $\left.A^{n}\right)$.

2.2. Remarks. Let $A$ be a commutative $k$-algebra.

1. The module of Kähler differential, $\Omega_{A / k}^{1}$, exists (see [21], [13]).

2. $\Lambda: \operatorname{Hom}_{A}\left(\Omega_{A / k}^{1}, M\right) \longrightarrow \mathcal{D} \operatorname{er}(A, M)$ defined by $\varphi \mapsto \varphi \circ d$ it is an isomorphism of $A$-modules. In particular,

$$
\operatorname{Hom}_{A}\left(\Omega_{A / k}^{1}, A\right) \cong \mathcal{D}_{\mathrm{er}}(A)
$$

$\left\{y_{1}, \ldots, y_{k}\right\}$ is a set of generators of the $k$-algebra $A$, then $\left\{d y_{1}, \ldots, d y_{k}\right\}$ is a set of generators of the $A$-module $\Omega_{A / k}^{1}$. Thus it is a finitely generated $A$-module, and therefore also finitely presented, since $A$ is a Noetherian ring. presented. In fact, since $A$ is $k$-algebra of finite type, we have that $A=S / I$, where $S=k\left[x_{1}, \ldots, x_{n}\right]$ is the 
polynomial algebra in $n$ variables and $I$ an ideal in $S$. So, follows from Lemma 2.1.2 in [3] that $\mathcal{D}_{\operatorname{er}}(S / I) \cong\left\{\partial \in \mathcal{D}_{\operatorname{er}}(S) \mid \partial(I) \subseteq I\right\} / I \mathcal{D}_{\operatorname{er}}(S)$, which is a submodule of $\mathcal{D}_{\mathrm{er}}(S) / I \mathcal{D}_{\mathrm{er}}(S) \cong(S / I)^{n}=A^{n}$. Since $A$ is Noetherian, it is a finitely generated $A$-module, and therefore also finitely presented.

3. If $\mathcal{D}_{\text {er }}(A)$ is a finitely generate $A$-module having $\left\{\partial_{1}, \ldots, \partial_{n}\right\}$ as a set of generators, then $\left\{\hat{\partial}_{1}, \ldots, \hat{\partial}_{n}\right\}$ generated $\operatorname{Hom}_{A}\left(\Omega_{A / k}^{1}, A\right)$ as $A$-module.

\subsection{Connection \& Covariant Derivative}

We define a connection on $M$ to be a $k$-linear homomorphism

$$
\nabla: M \longrightarrow \Omega_{A / k}^{1} \otimes_{A} M
$$

satisfying $\nabla(a m)=a \nabla(m)+d a \otimes m$, for all $a \in A$ and $m \in M(d$ is the universal derivation).

We denote by $\operatorname{End}_{k}(M)$ the vector space of $k$-linear endomorphisms of $M$. We will consider the notion of a covariant derivative on $M$, as an A-linear homomorphism

$$
\begin{aligned}
& \mathbf{D}: \mathcal{D}_{\text {er }}(A) \longrightarrow \operatorname{End}_{k}(M) \\
& \partial \quad \longmapsto \quad \mathbf{D}_{\partial}
\end{aligned}
$$

such that $\mathbf{D}_{\partial}(a m)=a \mathbf{D}_{\partial}(m)+\partial(a) m$ for every $a \in A$ and $m \in M$.

2.3. Proposition. Let $A$ be a commutative $k$-algebra. If the $A$-module $M$ has a connection, then $M$ has a covariant derivative.

Proof. Let $\nabla: M \longrightarrow \Omega_{A / k}^{1} \otimes_{A} M$ be a connection on $M$. Now, we use the universal property of tensor products to associate to each $\partial \in \mathcal{D} \operatorname{er}(A)$ an $A$ linear homomorphism $\widetilde{\partial}: \Omega_{A / k}^{1} \otimes_{A} M \longrightarrow M$ such that $\widetilde{\partial}(\delta \otimes m)=\hat{\partial}(\delta) m$, here $\hat{\partial}$ comes from the universal property of the Kähler differential module.

Now define $\mathbf{D}_{\partial}: M \rightarrow M$ by $\mathbf{D}_{\partial}=\widetilde{\partial} \circ \nabla$. An easy verification show that $\mathbf{D}_{\partial} \in \operatorname{End}_{k}(M)$ and that $\partial \mapsto \mathbf{D}_{\partial}$ defines a covariant derivative on $M$.

Thus we concluded easily that.

2.4. Corollary. Let $A$ be a commutative $k$-algebra. If the $A$-module $M$ does not admit a covariant derivative, then neither admit connections. 
So an important question here is to know what class of modules admit connections. For example, $\nabla:(t) \rightarrow \Omega_{[t] /}^{1} \otimes_{[t]}(t) \cong(t)$ given by the usual derivation $\frac{d}{d t}$ defines a connection on the non-projective $[t]$-module $(t)$. On the other hand, as we shall see in the next proposition, every projective module has a connection.

2.5. Proposition. Let $A$ be a commutative $k$-algebra and $M$ an $A$ module. Set $\operatorname{Con}(M)=\{\nabla \mid \nabla$ is a connection on $M\}$. Then

1. Every projective $A$-module admits a connection.

2. If $\nabla \in \mathcal{C}$ on $(M)$ and $L \in \operatorname{End}_{A}\left(M, \Omega_{A / k}^{1} \otimes_{A} M\right)$ then $\nabla+L \in \mathcal{C}$ on $(M)$.

3. If $\operatorname{Con}(M) \neq \emptyset$, then $\oplus: \mathcal{C}$ on $(M) \times \operatorname{End}_{A}\left(M, \Omega_{A / k}^{1} \otimes_{A} M\right) \rightarrow \mathcal{C}$ on $(M)$ given by $\nabla \oplus L=\nabla+L$ defines a right action of $\operatorname{End}_{A}\left(M, \Omega_{A / k}^{1} \otimes_{A} M\right)$ on $\mathcal{C}$ on $(M)$, which is free and transitive. Thus $\mathcal{C}$ on $(M)$ is an affine space. In particular, $\operatorname{End}_{A}\left(M, \Omega_{A / k}^{1} \otimes_{A} M\right)$ can be identified with $\operatorname{Con}(M)$.

Proof. We first established that free modules have a connection. If $F$ is a free $A$-module with basis $\left\{u_{j}\right\}_{j \in \mathcal{J}}$, then define $\nabla: F \longrightarrow \Omega_{A / k}^{1} \otimes_{A} F$ by $\nabla(m)=\sum_{i \in \mathcal{J}} d\left(a_{i}\right) \otimes u_{i}$, if $m=\sum_{i \in \mathcal{J}} a_{i} u_{i}\left(a_{i} \neq 0\right.$ only for a finite set of $i)$. It is easy to conclude that $\nabla$ is a connection on $F$.

Now, let $M$ be a projective $A$-module. Thus we can consider an $A$ module $N$ such that $M \times N$ is a free $A$-module. Hereafter, let $\nabla$ be a connection on $M \times N$ and define

$$
\widetilde{\nabla}: M \longrightarrow \Omega_{A / k}^{1} \otimes_{A} M \text { by } \widetilde{\nabla}(m)=p_{1}(\nabla(m, 0)),
$$

where $p_{1}$ is the projection from $\Omega_{A / k}^{1} \otimes_{A}(M \times N) \cong\left(\Omega_{A / k}^{1} \otimes_{A} M\right) \times\left(\Omega_{A / k}^{1} \otimes_{A}\right.$ $N)$ to $\Omega_{A / k}^{1} \otimes_{A} M$. It is a straightforward verification to show that $\widetilde{\nabla}$ is a connection on $M$.

The verification on second statement we left to the reader.

To prove the third statement, first note that $\nabla+L$ defines a connection on $M$ for every $\nabla \in \mathcal{C}$ on $(M)$ and $L \in \operatorname{End}_{A}\left(M, \Omega_{A / k}^{1}\right)$. Now, follows from the definitions that $\oplus$ defines a free right action of $\operatorname{End}_{A}\left(M, \Omega_{A / k}^{1} \otimes_{A} M\right)$ on $\mathcal{C}$ on $(M)$. To verify that this action is transitive, let us consider $\nabla, \nabla_{0} \in$ $\operatorname{Con}(M)$. Since $\operatorname{Con}(M) \subset \operatorname{Hom}_{k}\left(M, \Omega_{A / k}^{1} \otimes_{A} M\right)$, we get the $k$-linear homomorphism $\nabla-\nabla_{0}: M \rightarrow \Omega_{A / k}^{1} \otimes_{A} M$ given by $\left(\nabla-\nabla_{0}\right)(m)=$ 
$\nabla(m)-\nabla_{0}(m)$, if $m \in M$. So, now it is sufficient to prove that the $k^{-}$ linear homomorphism $\nabla-\nabla_{0}$ is also an $A$-linear homomorphism. In fact,

$$
\begin{aligned}
\left(\nabla-\nabla_{0}\right)(a m) & =\nabla(a m)-\nabla_{0}(a m) \\
& =a \nabla(m)-d a \otimes m-\left(a \nabla_{0}(m)-d a \otimes m\right) \\
& =a\left(\nabla-\nabla_{0}\right)(m) .
\end{aligned}
$$

Finally, fix $\nabla_{0} \in \mathcal{C}$ on $(M)$ and note that $L \mapsto \nabla_{0} \oplus L$ defined from $\operatorname{End}_{A}\left(M, \Omega_{A / k}^{1} \otimes_{A} M\right)$ onto $\mathcal{C}$ on $(M)$, it is a bijection.

\subsection{Example. The $\mathcal{C}_{\mathcal{U}}^{\infty}$-module $\Gamma(E)$ admits a connection}

Let $\mathcal{U}$ be a compact differentiable manifold and $\mathcal{C}_{\mathcal{U}}^{\infty}$ be the commutative $\mathbf{R}$-algebra of all infinitely differentiable real-valued functions on $\mathcal{U}$. For a differentiable (or smooth) vector bundle $E$ on $\mathcal{U}$, set $\Gamma(E)$ be the space of differentiable sections.

Next, we remember the correspondence established by Swan in [30] (details may be found in [27]) between

$\{$ vector bundles over cal $U\} \Longleftrightarrow\left\{\right.$ finitely generated projective $\mathcal{C}_{\mathcal{U}}^{\infty}$-modules $\}$.

In fact, it is proven that: $\Gamma(E)$ is a finitely generated projective $\mathcal{C}_{\mathcal{U}}^{\infty}$-module. Conversely, if $\mathcal{E}$ be a finitely generated projective $\mathcal{C}_{\mathcal{U}}^{\infty}$-module, then there exists a vector bundle $E$ such that $\mathcal{E} \cong \Gamma(E)$.

Since every projective module admits a connection (cf. Prop. 2.5) and consequently a covariant derivative (cf. Prop. 2.3). Then the $\mathcal{C}_{\mathcal{U}}^{\infty}$-module $\Gamma(E)$ admits a connection, as well as a covariant derivative.

\section{Some Counterexamples}

We consider the commutative $\mathbf{R}$-algebra $\mathcal{A}$ given by

$$
\mathcal{A}=\left\{c_{0}+\sum_{i=1}^{n} a_{i} x^{i}+\sum_{j=1}^{m} b_{j} y^{j} \mid n, m \in \mathbf{N}, c_{0}, a_{i}, b_{i} \in \mathbf{R} \text { and } x y=0\right\},
$$

with the usual operations of addition and multiplication on the polynomial ring. 
Since each $f$ in $\mathcal{A}$ can be written in a unique way as $f=f_{0}+f_{1}(x) x+$ $f_{2}(y) y$, then we define $\partial_{i}: \mathcal{A} \longrightarrow \mathcal{A}$ for $1 \leq i \leq 2$ by

$$
\partial_{1}(f)=x\left(f_{1}(x)+x f_{1}{ }^{\prime}(x)\right) \text { and } \partial_{2}(f)=y\left(f_{2}(y)+y f_{2}{ }^{\prime}(y)\right),
$$

here $f_{1}{ }^{\prime}$ and $f_{2}{ }^{\prime}$ denote the usual derivative of the polynomials $f_{1}$ and $f_{2}$, respectively.

3.1. Lemma. Let $\partial_{1}$ and $\partial_{2}$ as in (3.2). Then

1. $f \partial_{1}+g \partial_{2}=0 \Longleftrightarrow f=y a$ and $g=x b$ for some $a, b \in \mathcal{A}$. In particular, it is verified that $y \partial_{1}=0$ and $x \partial_{2}=0$.

2. $\partial_{1}$ and $\partial_{2} \in \mathcal{D}_{\operatorname{er}}(\mathcal{A})$ and $\operatorname{D} \operatorname{er}(\mathcal{A})=\mathcal{A} \partial_{1}+\mathcal{A} \partial_{2}$.

3. $\left\{\partial_{1}, \partial_{2}\right\}$ is a minimal set of generators of the $\mathcal{A}$-module $\mathcal{D}_{\mathrm{er}}(\mathcal{A})$.

4. If $\mathcal{N}=\left\{(\alpha y, \beta x) \in \mathcal{A}^{2} \mid \alpha, \beta \in \mathcal{A}\right\}$, then $(a, b)+\mathcal{N} \mapsto a \partial_{1}+b \partial_{2}$ defines an $\mathcal{A}$-linear isomorphism from $\mathcal{A}^{2} / \mathcal{N}$ to $\mathcal{D}_{\text {er }}(\mathcal{A})$.

Proof. The first statement follows from the definition of $\partial_{1}$ and $\partial_{2}$, together with the fact that $\mathcal{A}$ is a $\mathbf{R}$-vector space with basis

$\left\{1, x, \ldots, x^{n}, \ldots, y, \ldots, y^{m}, \ldots\right\}$. In fact, $f=y f_{2}(y)$ and $g=x g_{1}(x)$, if we write $f=f_{0}+f_{1}(x) x+f_{2}(y) y$ and $g=g_{0}+g_{1}(x) x+g_{2}(y) y$.

It is a straightforward verification to show that $\partial_{1}$ and $\partial_{2}$ belong to $\mathcal{D}_{\text {er }}(\mathcal{A})$. Now consider $\partial \in \mathcal{D}_{\operatorname{er}}(\mathcal{A})$ and set

$$
\begin{gathered}
\partial(x)=a_{0}+a_{1}(x) x+a_{2}(y) y \\
\partial(y)=b_{0}+b_{1}(x) x+b_{2}(y) y .
\end{gathered}
$$

Having in mind that $x y=0$, we concluded that $0=x \partial(y)+y \partial(x)$. Thus substituting (3.3) in the last equation, we get that

$$
\partial(x)=a_{1}(x) x \quad \text { and } \quad \partial(y)=b_{2}(y) y .
$$

Thus from (3.3) we obtain that $\partial=a_{1}(x) \partial_{1}+b_{2}(y) \partial_{2}$. Therefore $\mathcal{D} \operatorname{er}(\mathcal{A})=$ $\mathcal{A} \partial_{1}+\mathcal{A} \partial_{2}$.

Now suppose that $\partial$ is a generator of the $\mathcal{A}$-module $\mathcal{D}$ er $(\mathcal{A})$. Thus we have that

$$
\begin{aligned}
& \partial_{1}=\left(\alpha_{0}+\alpha_{1}(x) x+\alpha_{2}(y) y\right) \partial \\
& \partial_{2}=\left(\beta_{0}+\beta_{1}(x) x+\beta_{2}(y) y\right) \partial .
\end{aligned}
$$


We can write $\partial=p(x) \partial_{1}+q(y) \partial_{2}$ and substitute it in (3.5). Then keeping in mind that $y \partial_{1}=0=x \partial_{2}$ (cf. first statement), we conclude that

$$
\begin{aligned}
& \partial_{1}=p(x)\left(\alpha_{0}+\alpha_{1}(x) x\right) \partial_{1}+q(y)\left(\alpha_{0}+\alpha_{2}(y) y\right) \partial_{2} \\
& \partial_{2}=p(x)\left(\beta_{0}+\beta_{1}(x) x\right) \partial_{1}+q(y)\left(\beta_{0}+\beta_{2}(y) y\right) \partial_{2} .
\end{aligned}
$$

So evaluating the expression for $\partial_{2}$ in (3.6) at $y$, we get that $y=$ $q(y)\left(\beta_{0}+\beta_{2}(y) y\right) y$ which implies that $q(y) \neq 0$. Now let us to evaluate the expression for $\partial_{1}$ in (3.6) at $x$ and $y$, respectively. Then we obtain

$$
x=p(x)\left(\alpha_{0}+\alpha_{1}(x) x\right) x \quad \text { and } \quad 0=q(y)\left(\alpha_{0}+\alpha_{2}(y) y\right) y .
$$

Since $q(y) \neq 0$, then from the last equality above we get that $\alpha_{0}=$ $0=\alpha_{2}(y)$, thus the first equality in (3.7) becomes $x=p(x) \alpha_{1}(x) x^{2}$. The last equation implies that $1=p(x) \alpha_{1}(x) x$. Thus we have arrived at a contradiction. So the third statement is proved.

The last item follows from the other statements already proved in this lemma.

3.2. Lemma. Set $\mathcal{K}=\left\{(\alpha y, \alpha x) \in \mathcal{A}^{2} \mid \alpha \in \mathcal{A}\right\}$ and let $d_{\mathcal{A}}: \mathcal{A} \rightarrow \mathcal{A}^{2} / \mathcal{K}$ be defined by $a \mapsto\left(\frac{\partial a}{\partial x}, \frac{\partial a}{\partial y}\right)+\mathcal{K}$. Then we have that:

1. $d_{\mathcal{A}}$ is a derivation.

2. The pair $\left(\mathcal{A}^{2} / \mathcal{K}, d_{\mathcal{A}}\right)$ verifies the universal property of $\Omega_{\mathcal{A} / k}^{1}$. In particular, we conclude that $\Omega_{\mathcal{A} / k}^{1} \cong \mathcal{A}^{2} / \mathcal{K}$ as $\mathcal{A}$-modules.

3. The elements $\delta^{1}=d x$ and $\delta^{2}=d y\left(\right.$ in $\left.\Omega_{\mathcal{A} / k}^{1}\right)$ generated $\Omega_{\mathcal{A} / k}^{1}$ as an $A$-module. Moreover

(3.8) $f \delta^{1}+g \delta^{2}=0 \Longleftrightarrow f=\alpha y$ and $g=\alpha x$ for some $\alpha \in \mathcal{A}$.

4. $y \delta^{1} \neq 0$ and $x \delta^{2} \neq 0$.

Proof. It is a straightforward verification to show that $d_{\mathcal{A}}$ is a derivation. On the other hand, if $N$ is an $A$-module and $\partial \in \mathcal{D}$ er $(\mathcal{A}, N)$, then define $\varphi: \mathcal{A}^{2} \longrightarrow N$ by $\varphi(\alpha, \beta)=\alpha \partial(x)+\beta \partial(y)$. Note that $\varphi$ is an $\mathcal{A}$-linear homomorphism such that $\mathcal{K} \subset \operatorname{ker}(\varphi)$. So it induces an $\mathcal{A}$-linear homomorphism $\hat{\partial}$ from $\mathcal{A}^{2} / \mathcal{K}$ to $N$ such that $\hat{\partial}((\alpha, \beta)+\mathcal{K})=\varphi(\alpha, \beta)$. After an easy computation we conclude that $\hat{\partial}$ is the unique $\mathcal{A}$-linear homomorphism such that $\hat{\partial} \circ d_{\mathcal{A}}=\partial$. 
Now, observe that applying the universal property to the $\mathcal{A}$-module $\Omega_{\mathcal{A} / k}^{1}$ and $d$ (the universal derivation). We obtain an $\mathcal{A}$-linear isomorphism $\psi: \mathcal{A}^{2} / \mathcal{K} \longrightarrow \Omega_{\mathcal{A} / k}^{1}$ such that $(1,0)+\mathcal{K} \mapsto d x$ and $(0,1)+\mathcal{K} \mapsto d y$. From where we deduce easily the third statement.

Finally, let us assume that $y \delta^{1}=0$, so applying the inverse of the $\mathcal{A}_{-}$ linear isomorphism $\psi$ above, we obtain that $(y, 0)+\mathcal{K}=\mathcal{K}$ which implies that $y=\alpha y$ and $0=\alpha x$ for some $\alpha$ in $\mathcal{A}$. Writing $\alpha=\alpha_{0}+x \alpha_{1}(x)+y \alpha_{2}(y)$ we achieve that:

$$
y=\alpha_{0} y+\alpha_{2}(y) y^{2} \quad \text { and } \quad 0=\alpha_{0} x+\alpha_{1}(x) x^{2} .
$$

Since $\left\{1, x, x^{2}, \ldots, y, y^{2}, \ldots\right\}$ it is a basis of the $\mathbf{R}$-vector space $\mathcal{A}$, we conclude using the equations above that $\alpha_{0}=1$ and $\alpha_{0}=0$. Thus we have arrived to a contradiction. In a similar way we can show that $x \delta^{2} \neq 0$.

\subsection{A module without covariant derivative}

Let $\mathcal{A}$ be the $\mathbf{R}$-algebra defined in (3.1) and set $M=\Omega_{\mathcal{A} / k}^{1}$.

Let us suppose that there exists a covariant derivative $\mathbf{D}$ on $M$. For simplicity, we will use the notation $\mathbf{D}_{i}$ instead of $\mathbf{D}_{\partial_{i}}$ for $i=1,2$. Assume that:

$$
\mathbf{D}_{2} \delta^{1}=a \delta^{1}+b \delta^{2} \quad \text { for some } a, b \in \mathcal{A} .
$$

Since $x \partial_{2}=0$ (see Lemma 3.1), we obtain using (3.9) that

$$
0=\mathbf{D}_{x \partial_{2}} \delta^{1}=x \mathbf{D}_{2} \delta^{1}=x\left(a \delta^{1}+b \delta^{2}\right)=x a \delta^{1}+x b \delta^{2} .
$$

Then follows from (3.10) and (3.8) that $x a=\mu y$ for some $\mu \in \mathcal{A}$.

Writing $\mu=\mu_{0}+x \mu_{1}(x)+y \mu_{2}(y)$ we achieve that:

$$
x a=\left(\mu_{0}+y \mu_{2}(y)\right) y .
$$

Now setting $a=a_{0}+x a_{1}(x)+y a_{2}(y)$, follows from (3.11) that $a_{0}=0$.

Now have in mind one more time that $x \partial_{2}=0$, and $\partial_{2}(x)=0, \partial_{2}(y)=y$ (cf. (3.2)) to conclude from (3.8) and (3.9) that:

$$
\begin{aligned}
0=\mathbf{D}_{2}\left(y \delta^{1}+x \delta^{2}\right)=\mathbf{D}_{2}\left(y \delta^{1}\right)+\mathbf{D}_{2}\left(x \delta^{2}\right) & =y \mathbf{D}_{2}\left(\delta^{1}\right)+y \delta^{1}+x \mathbf{D}_{2}\left(\delta^{2}\right) \\
& =y(1+a) \delta^{1}+y b \delta^{2} .
\end{aligned}
$$

Then follows from (3.8) that

$$
y(1+a)=\nu y \quad \text { and } \quad y b=\nu x \quad \text { for some } \quad \nu \in \mathcal{A} .
$$


Now writing $\nu=\nu_{0}+x \nu_{1}(x)+y \nu_{2}(y)$, we get

$$
y(1+a)=\left(\nu_{0}+y \nu_{2}(y)\right) y \quad \text { and } \quad y b=\left(\nu_{0}+x \nu_{1}(x)\right) x .
$$

Thus follows from the second equality in (3.12) that $\nu_{0}=0$ and consequently that $1+a_{0}=0$, i.e. $a_{0}=-1$ (after substitution of $\nu_{0}=0$ in the first equality in (3.12)). Thus we have arrived at a contradiction.

\subsection{A module with covariant derivative and without connection}

Let $\mathcal{A}$ be the commutative $\mathbf{R}$-algebra defined in (3.1).

- The $\mathcal{A}$-module $M=\mathcal{D}_{\text {er }}(A)$ has a covariant derivative.

Define $\varphi_{i}: \mathcal{A}^{2} \longrightarrow M$ for $i=1,2$ by $\varphi_{i}(a, b)=\partial_{i}(a) \partial_{1}+\partial_{i}(b) \partial_{2}$ (with $\partial_{1}$ and $\partial_{2}$ as in (3.2)). Note that each $\varphi_{i}$ is an $\mathbf{R}$-linear homomorphism and that $\mathcal{N}$ is contained in the kernel of both homomorphisms. Thus they induce $\mathbf{R}$-linear endomorphisms from $\mathcal{A}^{2} / \mathcal{N}$ to $M$, which (keeping in mind the isomorphism in lemma 3.1) determine the $\mathbf{R}$-linear endomorphisms $\mathbf{D}_{i} \in \operatorname{End}_{\mathbf{R}}(M)$ such that

$$
\mathbf{D}_{i}\left(a \partial_{1}+b \partial_{2}\right)=\partial_{i}(a) \partial_{1}+\partial_{i}(b) \partial_{2} \quad i=1,2 .
$$

Now we consider $\varphi: \mathcal{A}^{2} \longrightarrow \operatorname{End}_{\mathbf{R}}(M)$ defined by $\varphi(a, b)=a \mathbf{D}_{1}+$ $b \mathbf{D}_{2}$. Note that $\varphi$ is an $A$-linear homomorphism such that $\mathcal{N} \subset \operatorname{ker}(\varphi)$ (since $y \mathbf{D}_{1}=x \mathbf{D}_{2}=0$ ). Thus, using again the isomorphism in lemma 3.1, we conclude that $\varphi$ induces an $A$-linear homomorphism $\mathbf{D}: \mathcal{D} \operatorname{er}(A) \longrightarrow$ $\operatorname{End}_{k}(M)$ such that $\mathbf{D}_{a \partial_{1}+b \partial_{2}}=a \mathbf{D}_{1}+b \mathbf{D}_{2}$.

Now, an easy verification shows that $\mathbf{D}_{\partial_{i}}(a m)=a \mathbf{D}_{\partial_{i}}(m)+\partial_{i}(a) m$ for any $a \in \mathcal{A}$ and $m \in M$. Having in mind that $\mathbf{D}_{a \partial_{1}+b \partial_{2}}=a \mathbf{D}_{1}+b \mathbf{D}_{2}$ and doing some more computations, we concluded that $\mathbf{D}$ is a covariant derivative on $M=\mathcal{D}_{\operatorname{er}}(A)$.

- The $\mathcal{A}$-module $M=\mathcal{D}_{\mathrm{er}}(A)$ does not have a connection.

Suppose that $M$ admits a connection $\nabla$. Thus we can write

$$
\nabla\left(\partial_{1}\right)=\sum_{i, j=1}^{2} a_{i j} \delta^{i} \otimes \partial_{j}
$$

with $\delta^{i}(i=1,2)$ as in Lemma 3.2 and $a_{i j} \in \mathcal{A}$.

Now remember that $y \partial_{1}=0$ (cf. lemma 3.1). Therefore

$$
0=\nabla\left(y \partial_{1}\right)=y \nabla\left(\partial_{1}\right)+\delta^{2} \otimes \partial_{1} .
$$


Furthermore, if we multiply both sides of Eq. (3.13) by $y$ (and have in mind that $\left.y \partial_{1}=0\right)$, we get:

$$
y \nabla\left(\partial_{1}\right)=a_{12} y \delta^{1} \otimes \partial_{2}+a_{22} y \delta^{2} \otimes \partial_{2} .
$$

Since $y \delta^{1}+x \delta^{2}=0$ (cf. third statement in Lemma 3.2), after substitution Eq. (3.15) becomes

$$
y \nabla\left(\partial_{1}\right)=\left(a_{22} y-a_{12} x\right) \delta^{2} \otimes \partial_{2} .
$$

Substituting (3.16) in Eq. (3.14), we arrive to equation:

$$
0=\left(a_{22} y-a_{12} x\right) \delta^{2} \otimes \partial_{2}+\delta^{2} \otimes \partial_{1} .
$$

On the other hand, since $\left\{\partial_{1}, \partial_{2}\right\}$ is a set of generators of the $\mathcal{A}$-module $\mathcal{D}_{\text {er }}(\mathcal{A})$, then $\left\{\hat{\partial}_{1}, \hat{\partial}_{2}\right\}$ it is a set of generators of $\operatorname{Hom}_{A}\left(\Omega_{\mathcal{A} / k}^{1}, \mathcal{A}\right)$ as an $\mathcal{A}^{-}$ module, which verified that

$$
\hat{\partial}_{1}\left(\delta^{1}\right)=x, \hat{\partial}_{1}\left(\delta^{2}\right)=0, \hat{\partial}_{2}\left(\delta^{1}\right)=0 \text { and } \hat{\partial}_{2}\left(\delta^{2}\right)=y
$$

Next, we apply the universal property of tensor products to get the $\mathcal{A}$-linear homomorphism $\phi: \Omega_{\mathcal{A} / k}^{1} \otimes_{\mathcal{A}} \mathcal{D}_{\mathrm{er}}(\mathcal{A}) \longrightarrow \operatorname{End}_{\mathcal{A}}\left(\Omega_{\mathcal{A} / k}^{1}\right)$ such that $\delta^{i} \otimes \partial_{j} \mapsto \phi_{i j}$ with $\phi_{i j}\left(\delta^{k}\right)=\hat{\partial}_{j}\left(\delta^{k}\right) \delta^{i}$ for any $i, j, k \in\{1,2\}$.

Now applying $\phi$ to Eq. (3.17), we obtain:

$$
0=\left(a_{22} y-a_{12} x\right) \phi_{22}+\phi_{21} .
$$

So evaluating the homomorphism in (3.19) at $\delta^{1}$, we get (using (3.18) that $0=x \delta^{2}$. Now, the last statement in lemma 3.2 assures us that $x \delta^{2} \neq 0$. Thus we have arrived at a contradiction.

\section{Equivalent notions and obstruction}

Eriksen has proven in Proposition 4.7 (at p. 51 in [18]) that the notions of connections and covariant derivative are equivalent when we consider regular $k$-algebras of finite type. For the reader's convenience, we review Eriksen's proof giving a few more details in the next proposition.

4.1. Lemma. Let $A$ be a commutative $k$-algebra of finite type then $\Omega_{A / k}^{1}$ and $\mathcal{D}_{\mathrm{er}}(A)$ are finitely presented $A$-modules. 
Proof. We first prove that $\Omega_{A / k}^{1}$ is a finitely presented $A$-module. In fact, if $\left\{y_{1}, \ldots, y_{k}\right\}$ is a set of generators of the $k$-algebra $A$, then $\left\{d y_{1}, \ldots, d y_{k}\right\}$ is a set of generators of the $A$-module $\Omega_{A / k}^{1}$. Thus it is a finitely generated $A$-module, and therefore also finitely presented, since $A$ is a Noetherian ring.

In order to prove the second statement, we recall that $A=S / I$, for some polynomial algebra $S=k\left[x_{1}, \ldots, x_{n}\right]$ in $n$ variables and some ideal $I$ in $S$, since $A$ is $k$-algebra of finite type. So, follows from Lemma 2.1.2 in [3] that $\mathcal{D}_{\mathrm{er}}(S / I) \cong\left\{\partial \in \mathcal{D}_{\mathrm{er}}(S) \mid \partial(I) \subseteq I\right\} / I \mathcal{D}_{\mathrm{er}}(S)$, which is a submodule of $\mathcal{D}_{\text {er }}(S) / I \mathcal{D}_{\text {er }}(S) \cong(S / I)^{n}=A^{n}$. Since $A$ is Noetherian, it is a finitely generated $A$-module, and therefore also finitely presented.

4.2. Proposition. Let $A$ be a regular, commutative $k$-algebra of finite type. Then, every covariant derivative induces a connection. In fact, this establishes a correspondence between the notions of connection and covariant derivative.

Proof. Having in mind the isomorphism in (2.1) which associated to each derivation $\partial \in \mathcal{D}_{\operatorname{er}}(A)$ the $A$-linear homomorphism $\hat{\partial}: \Omega_{A / k}^{1} \rightarrow A$ such that $\partial=\hat{\partial} \circ d$. Next, we use the universal property of tensor products to conclude that there exists a unique $A$-linear homomorphism

$$
\Psi: \Omega_{A / k}^{1} \otimes_{A} M \longrightarrow H_{o m}\left(\mathcal{D}_{\mathrm{er}}(A), M\right) \text { such that } \Psi(\delta \otimes m)(\partial)=\hat{\partial}(\delta) m .
$$

We propose in what follows to show that $\Psi$ is an isomorphism. In order to do that, we are going to verify that, $\Psi_{p}$, the localization of $\Psi$ at the prime ideal $p$ of $A$ is an isomorphism for any prime ideal in $A$.

We begin by noting that:

$$
\begin{aligned}
\left(\Omega_{A / k}^{1} \otimes_{A} M\right)_{p} & \cong\left(\Omega_{A / k}^{1}\right)_{p} \otimes_{A_{p}} M_{p} & & \text { (See Proposition } 3.7 \mathrm{in} \mathrm{[1])} \\
& \cong \Omega_{A_{p} / k}^{1} \otimes_{A_{p}} M_{p} & & \text { (See Proposition 16.9 in [13]) } \\
& \cong A_{p}^{n_{p}} \otimes_{A_{p}} M_{p} \cong M_{p}^{n_{p}} & & \text { (See Theorem 8.8 in [21]) }
\end{aligned}
$$

To arrive to the isomorphism in the last line above, we use the hypothesis of regularity of $A$ and denote by $n_{p}$ the Krull dimension of $A_{p}$.

Now, Prop. 16.9 (in [13]) assured us that $\left(\Omega_{A / k}^{1}\right)_{p} \cong \Omega_{A_{p} / k}^{1}$ as $A_{p}-$ modules. So, keeping in mind that $\Omega_{A / k}^{1}$ is an $A$-module finitely presented, we can apply Prop. 2.10 (in [13]) to concluded that $\left(\mathcal{D}_{\operatorname{er}}(A)\right)_{p} \cong$ $\left(\operatorname{Hom}_{A}\left(\Omega_{A / k}^{1}, A\right)\right)_{p} \cong \operatorname{Hom}_{A_{p}}\left(\left(\Omega_{A / k}^{1}\right)_{p}, A_{p}\right) \cong \operatorname{Hom}_{A_{p}}\left(\Omega_{A_{p} / k}^{1}, A_{p}\right)$. Now, 
follows from the universal property of the module of Kähler differentials, $\Omega_{A_{p} / k}^{1}$, that $\left(\mathcal{D}_{\text {er }}(A)\right)_{p} \cong \mathcal{D}_{\text {er }}\left(A_{p}\right)$ as $A_{p}$-modules. Moreover, using again the regularity of $A$, we have:

$$
\mathcal{D}_{\mathrm{er}}\left(A_{p}\right) \cong \operatorname{Hom}_{A_{p}}\left(\Omega_{A_{p} / k}^{1}, A_{p}\right) \cong \operatorname{Hom}_{A_{p}}\left(A_{p}^{n_{p}}, A_{p}\right) \cong A_{p}^{n_{p}} .
$$

Thus from (4.2) we have

$$
\operatorname{Hom}_{A_{p}}\left(\mathcal{D}_{\text {er }}\left(A_{p}\right), M_{p}\right) \cong \operatorname{Hom}_{A_{p}}\left(A_{p}^{n_{p}}, M_{p}\right) \cong\left(\operatorname{Hom}_{A_{p}}\left(A_{p}, M_{p}\right)\right)^{n_{p}} \cong M_{p}^{n_{p}} .
$$

Now, having in mind that $\left(\mathcal{D}_{\text {er }}(A)\right)_{p} \cong \mathcal{D}_{\text {er }}\left(A_{p}\right)$ as $A_{p}$-modules, and that $\mathcal{D}_{\text {er }}(A)$ is also an $A$-module finitely presented, we can apply one more time Prop. 2.10 (in [13]) and (4.3) to get:

$$
\left(\operatorname{Hom}_{A}\left(\mathcal{D}_{\operatorname{er}}(A), M\right)\right)_{p} \cong \operatorname{Hom}_{A_{p}}\left(\left(\mathcal{D}_{\mathrm{er}}(A)\right)_{p}, M_{p}\right) \cong M_{p}^{n_{p}} .
$$

Now let us consider $\left\{\delta^{1}, \ldots, \delta^{n_{p}}\right\}$ be a basis of the free $A_{p}$-module $\Omega_{A_{p} / k}^{1}$ and $\left\{\partial_{1}, \ldots, \partial_{n_{p}}\right\}$ be a basis of the free $A_{p}$-module $\mathcal{D}_{\text {er }}\left(A_{p}\right)$.

Right now, if $a_{1}, \ldots, a_{n_{p}}$ are the unique elements in $A_{p}$ such that $d\left(\frac{a}{1}\right)=$ $\sum_{j=1}^{n_{p}} a_{j} \delta^{j}$ (here $d$ is the universal derivation for $\Omega_{A_{p} / k}^{1}$ ). Then follows from the isomorphisms in (4.1) that the element $\frac{d a}{1} \otimes \frac{m}{1}$ is taken in $\left(a_{1} \frac{m}{1}, \ldots, a_{n_{p}} \frac{m}{1}\right)$.

Hereafter follows from the isomorphism in (4.4) that the element $\Psi_{p}\left(\frac{d a}{1} \otimes\right.$ $\left.\frac{m}{1}\right)$ determine an $A_{p}$-linear homomorphism such that $\partial_{i} \mapsto \partial_{i}\left(\frac{a}{1}\right) \frac{m}{1}=$ $\sum_{j=1}^{n_{p}} \hat{\partial}_{i}\left(\delta^{j}\right) a_{j} \frac{m}{1}$ for $i=1, \ldots, n_{p}$ (have in mind that $\partial_{i}(\alpha)=\hat{\partial}_{i}(d \alpha)$ for every $\left.\alpha \in A_{p}\right)$.

Thus $\Psi_{p}: M_{p}^{n_{p}} \longrightarrow M_{p}^{n_{p}}$ is given by

$$
\Psi_{p}\left(m_{1}, \ldots, m_{n_{p}}\right)=\left(\sum_{i=1}^{n_{p}} \hat{\partial}_{1}\left(\delta^{i}\right) m_{i}, \ldots, \sum_{i=1}^{n_{p}} \hat{\partial}_{n_{p}}\left(\delta^{i}\right) m_{i}\right) .
$$

Finally, note that the isomorphism in (4.2) takes $\partial_{i}$ to $\mathrm{v}_{i}=\left(\hat{\partial}_{i}\left(\delta^{1}\right), \ldots, \hat{\partial}_{i}\left(\delta^{n_{p}}\right)\right)$. So $\left\{\mathrm{v}_{1}, \ldots, \mathrm{v}_{n_{p}}\right\}$ is a basis of the free $A_{p}$-module $A_{p}^{n_{p}}$, so $\left(\hat{\partial}_{i}\left(\delta^{j}\right)\right)$ is a change of basis matrix of the free $A_{p}$-module $A_{p}^{n_{p}}$, which allows to conclude that $\Psi_{p}$ is an isomorphism for every prime ideal $p$ of $A$. Therefore $\Psi$ is an isomorphism.

Now let $\mathbf{D}: \mathcal{D}_{\mathrm{er}}(A) \longrightarrow \operatorname{End}_{k}(M)$ be a given covariant derivative on $M$. For each $m \in M$ define $\phi_{m}: \mathcal{D}_{\operatorname{er}}(A) \rightarrow M$ by $\partial \mapsto \mathbf{D}_{\partial}(m)$. A straightforward verification shows that $\phi_{m}$ is an $A$-linear homomorphism.

Next define $\nabla: M \longrightarrow \Omega_{A / k}^{1} \otimes_{A} M$ by $\nabla(m)=\Psi^{-1}\left(\phi_{m}\right)$. We leave to the reader the task of verifying that $\nabla$ is a connection on $M$. 


\subsection{Hochschild cohomology and obstruction}

Let $A$ be a commutative $k$-algebra and let $Q$ be an $A$-bimodule. We define Hochschild-cohomology on $A$ with values in $Q$ as the cohomology of the following complex:

Let $C^{n}(A, Q)=H_{0 m}\left(\otimes_{k}^{n} A, Q\right)$ for all $n \geq 0$, thus $C^{0}(A, Q) \equiv Q$ (since $\left.\otimes_{k}^{0} A=k\right), C^{1}(A, Q)=H_{k}(A, Q), C^{2}(A, Q)=H_{k}\left(A \otimes_{k} A, Q\right)$ and so on. Let us also consider the coboundary operators $d^{n}: C^{n}(A, Q) \longrightarrow$ $C^{n+1}(A, Q)$ be given by

$$
\begin{aligned}
d^{n}(\varphi)\left(a_{1} \otimes \cdots \otimes a_{n+1}\right) & =a_{1} \varphi\left(a_{2} \otimes \cdots \otimes a_{n+1}\right) \\
& +\sum_{i=1}^{n}(-1)^{i} \varphi\left(a_{1} \otimes \cdots \otimes a_{i} a_{i+1} \otimes \cdots \otimes a_{n+1}\right) \\
& +(-1)^{n+1} \varphi\left(a_{1} \otimes \cdots \otimes a_{n}\right) a_{n+1} .
\end{aligned}
$$

$H H^{n}(A, Q)=\operatorname{ker}\left(d^{n}\right) / \operatorname{Im}\left(d^{n-1}\right)$ (have in mind that $\operatorname{Im}\left(d^{-1}\right)=0$ ) will denote the $n$th Hochschild cohomology.

In fact, $d^{0}: Q \longrightarrow \operatorname{Hom}_{k}(A, Q)$ is given by $d^{0}(q)(a)=a q-q a$, so an easy verification shows that $\operatorname{Im}\left(d^{0}\right) \subset \mathcal{D}_{\mathrm{er}}(A, Q)$. Also, we have that $d^{1}$ : $\operatorname{Hom}_{k}(A, Q) \longrightarrow \operatorname{Hom}_{k}\left(A \otimes_{k} A, Q\right)$ is given by $d^{1}(\varphi)\left(a_{1} \otimes a_{2}\right)=a_{1} \varphi\left(a_{2}\right)-$ $\varphi\left(a_{1} a_{2}\right)+\varphi\left(a_{1}\right) a_{2}$, so $\operatorname{ker}\left(d^{1}\right)=\mathcal{D}_{\operatorname{er}}(A, Q)$. Therefore, $H H^{0}(A, Q)=\{q \in$ $Q \mid a q=q a \forall a \in A\}$ and $H H^{1}(A, Q)=\mathcal{D}_{\text {er }}(A, Q) / \operatorname{Im}\left(d^{0}\right)$ (the elements in $\operatorname{Im}\left(d^{0}\right)$ are usually named inner derivations $)$.

Thus, we have the following exact sequence:

$$
0 \rightarrow H H^{0}(A, Q) \rightarrow Q \stackrel{d^{0}}{\rightarrow} \mathcal{D}_{\operatorname{er}}(A, Q) \rightarrow H H^{1}(A, Q) \rightarrow 0
$$

Let us now consider $Q=H_{k}(N, P)$. Note that $H H^{0}\left(A, H o m_{k}(N, P)\right)=$ $\operatorname{Hom}_{A}(N, P)$ and $H H^{1}\left(A, \operatorname{Hom}_{k}(N, P)\right) \simeq \operatorname{Ext}_{A}^{1}(N, P)$ (the last isomorphism is proved at p. 170 in [7]), and the exact sequence (4.5) takes the form:

$0 \rightarrow \operatorname{Hom}_{A}(N, P) \rightarrow \operatorname{Hom}_{k}(N, P) \stackrel{d^{0}}{\rightarrow} \mathcal{D}_{\mathrm{er}}\left(A, \operatorname{Hom}_{k}(N, P)\right) \stackrel{\sigma}{\rightarrow} \operatorname{Ext}_{A}^{1}(N, P) \rightarrow 0$

The next proposition was proved by Eriksen (at p. 5 in [17]). In fact, we bring out the part what we are interested in.

4.3. Proposition. There exists an obstruction $c(M) \in \operatorname{Ext}_{A}^{1}\left(M, \Omega_{A / k}^{1} \otimes_{A}\right.$ $M)$, which is canonical and has the property that:

There exists a connection on $M$ if and only if $c(M)=0$.

$c(M)$ is called the Atiyah-Kodaira-Spencer class of $M$. 
Proof. Let us consider $c: A \rightarrow \operatorname{Hom}_{k}\left(M, \Omega_{A / k}^{1} \otimes_{A} M\right)$ given by $c(a)(m)=d a \otimes m$ where $d$ is the universal derivation. It's verified that $c \in \mathcal{D}_{\mathrm{er}}\left(A, H_{\text {om }}\left(M, \Omega_{A / k}^{1} \otimes_{A} M\right)\right)$. Now makes $N=M$ and $P=\Omega_{A / k}^{1} \otimes_{A}$ $M$ in (4.6) and let $c(M)=\sigma(c)$. Note that, if there is a connection $\nabla$ on $M$ then $\nabla \in \operatorname{Hom}_{k}\left(M, \Omega_{A / k}^{1} \otimes_{A} M\right)$ and $d^{0}(\nabla)=c$, which allows to conclude that $c(M)=\sigma(c)=\sigma\left(d^{0}(\nabla)\right)=0$ (from the exactness in (4.6)). Reciprocally, if $c(M)=0$ then $\sigma(c)=0$, so again from the exactness in (4.6), we have that there exists $\omega \in \operatorname{Hom}_{k}\left(M, \Omega_{A / k}^{1} \otimes_{A} M\right)$ such that $d^{0}(\omega)=c$, so $\omega$ is a connection on $M$.

\section{Connections defined via noncommutative differentials}

The result listed here can be found in Krähmer's Lecture 1 [25] and [29].

Here we considerer a $k$-algebra $A$ not necessarily commutative. Now, if $\Omega$ is an $A$-bimodule, then we say that $\partial: A \rightarrow \Omega$ is a derivation, if $\partial(a b)=a \partial(b)+\partial(a) b$ for any $a, b \in A($ since $\partial(r)=0$ for $r \in k$, easily we conclude that $\partial$ is $k$-linear).

Next we consider $A \otimes_{k} A$ as an $A$-bimodule (with the $A$-actions given by right multiplication in the second tensor component).

Let $\mu: A \otimes_{k} A \rightarrow A$ the multiplication map determined by $a \otimes b \mapsto a b$. Note that $\mu$ is an homomorphism of $A$-bimodules, so $\Omega^{1}:=\operatorname{ker}(\mu)$ is an $A$-bimodule. Note that

$$
d: A \rightarrow \Omega^{1} \text { given by } d(a)=1 \otimes a-a \otimes 1
$$

is a derivation.

Krähmer proved in [25] that:

5.1. Proposition. Notation as above. It is verified that:

1. $\{d a \mid a \in A\}$ generated $\Omega^{1}$ as an $A$-bimodule.

2. $\left(\Omega^{1}, d\right)$ satisfied the following universal property: If $L$ is any $A$ bimodule and $\partial: A \rightarrow L$ is a derivation with $\partial(k)=0$, then $\partial$ factors uniquely through $d$, that is, there is a unique $A$-bimodule homomorphism $\hat{\partial}: \Omega^{1} \rightarrow L$ such that $\partial=\hat{\partial} \circ d$.

We define a connection on $M$ to be an $k$-linear homomorphism

$$
\nabla: M \longrightarrow \Omega^{1} \otimes_{A} M
$$


satisfying $\nabla(a m)=a \nabla(m)+d a \otimes m$, for all $a \in A$ and $m \in M(d$ as in (5.1)).

Krähmer also established the equivalence between

\{ projective $A$-modules $\} \Longleftrightarrow\{A$-modules that admit connection $\}$.

5.2. Theorem. The connections on an $A$-module $M$ correspond bijectively to $A$-linear splittings of the action map $A \otimes_{k} M \rightarrow A(a \otimes m \mapsto a m)$. In particular, $M$ admits a connection if and only if it is projective.

Proof. See the Theorem at p. 13 in [25] or Corollary 3.4 in [29].

\subsection{The motivation for considering noncommutative differentials}

One of the major advances of science in the 20th century was the discovery of a mathematical formulation of quantum mechanics by Heisenberg in 1925. From a mathematical point of view, transition from classical mechanics to quantum mechanics amounts to, among other things, passing from the commutative algebra of classical observables to the noncommutative algebra of quantum mechanical observables. Recall that in classical mechanics an observable (e.g. energy, position, momentum, etc.) is a function on a manifold called the phase space of the system. Immediately after Heisenbergs work, ensuing papers by Dirac [12] and Born-Heisenberg-Jordan [2], made it clear that a quantum mechanical observable is a (selfadjoint) operator on a Hilbert space called the state space of the system. Thus the commutative algebra of functions on a space is replaced by the noncommutative algebra of operators on a Hilbert space.

In the early 80's, Alain Connes ([9], [10]) realized that a similar procedure can in fact be applied to areas of mathematics where the classical notions of space (e.g. measure space, or a smooth space) can not be used and can be replaced by a new idea of space, represented by a noncommutative algebra. His motivation can from the correspondence:

$\{$ Compact Haudorff spaces $\} \Longleftrightarrow\left\{\right.$ commutative $\mathcal{C}^{*}$-algebras $\} \quad X \mapsto \mathcal{C}_{X}$.

Which was established in the late 40's by the two Russian mathematicians Gelfand and Naimark (in [19]).

So when Noncommutative Geometry meets General Relativity a natural question arises about a satisfactory generalization of the notion of a linear connection. In order to do that Connes ([9]) has defined connections on 
modules using noncommutative differential forms. After this notion was extend to bimodules by Cuntz-Quillen in [11].

5.3. Remark. The seed of the notion of connection coming from 1869 paper by Christofell ([8]) has grown to be a great tree. One of his latest fruit appear in theoretical physics around 1964 and reappear in the real word at CERN as the Higgs boson of the Standard Model on July 2012 (see [16]).

Acknowledgements We thank to Eivind Eriksen (BI Norwegian Business School), Ulrich Krämer (University of Glasgow) and Wolmer Vasconcelos (Rutgers University) for their continuous advice.

\section{References}

[1] M. F. Atiyah and I. G. Macdonald, Introduction to Commutative Algebra. Addison-Wesley, Reading, MA, (1969).

[2] M. Born, W. Heisenberg, and P. Jordan, On quantum mechanics II, Zs. Phys. 35, pp. 557-615, (1926).

[3] P. Brumatti and A. Simis, The Module of Derivations of a StanleyReisner Ring, Proc. Amer. Math. Soc., Vol. 23, No. 5, pp. 1309-1318, (1995).

[4] M. P. do Carmo, Riemannian Geometry. Birkhäuser Boston, (1992).

[5] E. Cartan, Les espaces à connexion conforme, Ann. Soc. Polon. Math., Vol. 2, pp. 171-221, (1924).

[6] E. Cartan, Sur les variétés à connexion projective, Bull. Soc. Math. France, Vol. 52, pp. 205-241, (1924).

[7] E. Cartan and S. Eilenberg, Homological Algebra, Princeton University Press, (1956).

[8] E. B. Christoffel, Über die Transformation der homogenen Differentialausdrücke zweiten Grades, Journal für die reine u. angew. Math. (Crelle) 70, 46-70; Ges. math. Abh. I, 352-377, (1869). 
[9] A. Connes, Non-commutative differential geometry, Inst. Hautes Etudes Sci. Publ. Math. 62, pp. 257-360, (1985).

[10] A. Connes, Non-commutative geometry, Academic Press, (1994).

[11] J.Cuntz and D.Quillen, Algebra Extensions and Nonsingularity, J. Amer. Math. Soc. 8 (2), pp. 251-89, (1995).

[12] P. Dirac, The fundamental equations of quantum mechanics, Proc. Roy. Soc. A 109, pp. 642-653, (1926).

[13] D. Eisenbud, Commutative Algebra with a View Toward Algebraic Geometry, Graduate Texts in Math. 150, Springer, New York, (1995).

[14] C. Ehresmann, Sur les espaces fibrés associés à une variété différentiable, C. R. Acad. Sc. t. 216, pp. 628-630, (1943).

[15] C. Ehresmann, Les connexions infinitésimales dans un espace fibré diffrentiable, Colloque de Toplogie, CBRM, Bruxelles, pp. 29-55, (1950).

[16] J. Ellis, M. K. Gaillard and D. V. Nanopoulos, A Historical Profile of the Higgs Boson, arXiv:1201.6045.

[17] E. Eriksen, Connections and monodromy on modules, Technical Report, University of Oslo, (1995).

[18] E. Eriksen, Graded D-modules over Monomial Curves, Ph. D. thesis, University of Oslo, (2000).

[19] I. M. Gelfand and M. A. Naimark, On the imbedding of normed rings into the ring of operators on a Hilbert space, Math. Sbornik 12 (2), pp. 197-217, (1943).

[20] R. Gomes, Conexões e Curvatura: Uma abordagem algébrica, Dissertação de mestrado, DM-UFPE, (2009).

[21] R. Hartshorne, Algebraic Geometry. Graduate Texts in Math. 52, Springer, New York, (1977).

[22] M. L. Henrique,Derivações e Campos de Vetores, Dissertação de mestrado, DM-UFPE, (2001).

[23] N. Ferreira, Conexões e Transporte Paralelo: Abordagem Computacional, Dissertação de mestrado, DM-UFPE, (2010). 
[24] J. L. Koszul, Homologie et cohomologie des algebres de Lie, Bull. Soc. Math. France, Vol. 78, pp. 65-127, (1950).

[25] U. Krähmer, Dirac Operators, Lecture 1: Projective Modules and Connections, IPM Tehran 19, (2009).

[26] T. Levi-Civita and G. Ricci, Méthodes de calcul diffrential absolu et leurs applications, Math. Ann. B. 54, pp. 125-201, (1901).

[27] J. Nestruev, Smooth manifolds and observables. Graduate Texts in Math. 220, Springer-Verlag, New York, (2003).

[28] J. Rojas, O Funcional de Yang-Mills, Dissertação de mestrado, DMUFPE, (1990).

[29] R. B. da Silva, Existência de conexões versus módulos projetivos, Dissertação de mestrado, DM-UFPB, (2013).

[30] R. G. Swan, Vector Bundles and Projective Modules, Trans. Amer. Math. Soc. 105 (2), pp. 264-277, (1962).

\author{
Jacqueline Rojas \\ Departamento de Matemática \\ Universidade Federal da Paraiba (UFPB) \\ Cidade Universitária \\ 58051-900, João Pessoa - PB \\ Brasil \\ e-mail : jacq@mat.ufpb.br \\ and \\ Ramón Mendoza \\ CCEN - Departamento de Matemática \\ Universidade Federal de Pernambuco (UFPE) \\ Cidade Universitária, \\ 50740-540, Recife - PE \\ Brasil \\ e-mail : ramon@dmat.ufpe.br
}

